\title{
Das Signalisieren von Kohärenzstrukturen in deutschsprachigen wissenschaftlichen Texten
}

\author{
Helmut Gruber (Wien)
}

\begin{abstract}
This paper discusses the relation between newer approaches of intercultural rhetoric research (and specifically its distinction between "big" and "small" cultures) and the academic literacies approach which assumes that local institutional and individual biographic factors influence decisively the development of an individual academic writing competence. Based on this discussion it claims that the "research article" does not constitute a single genre but rather a genre colony consisting of members (genres) which differ according to linguistic, disciplinary, institutional and individual affiliations and status of writers and which are connected through a system of family resemblances. This claim is exemplified through a qualitative case study of two German academic texts, a "professional" published paper and a students' seminar paper. By applying a multi-level text analysis combining genre analysis and rhetorical structure analysis, it is shown that although the two texts exhibit several similarities in terms of their genre structure, their rhetorical macro- and micro-structures differ significantly. These results are interpreted as a corollary of the different institutional and hierarchical positions of the authors and as a single-case support for the initial claim of the paper.
\end{abstract}

\section{$1 \quad$ Einleitung}

Wissenschaftliche Aufsätze ('research articles') sind die wohl am meisten untersuchte Textsorte im Bereich der Wissenschaftskommunikation. Dabei ist nicht einmal ganz klar, ob es sich hier im engeren Sinne um eine „Textsorte“ handelt oder um eine ganze Gruppe von Textsorten (eine „Textsortenkolonie“ i.S. von Bhatia 2004) deren Struktur je nach Disziplin, (Wissenschafts-)Kultur, dem Ausmaß der fachlichen Sozialisation des Autors/der Autorin und anderen Einflussfaktoren variiert. Denn sowohl Untersuchungen von wissenschaftlichen Aufsätzen in verschiedenen Disziplinen (z. B. Gray 2011) wie auch Untersuchungen wissenschaftlicher Aufsätze aus verschiedenen (Wissenschafts-)Kulturen zeigen zum Teil starke Unterschiede zwischen den Texten, die unter dieser Bezeichnung veröffentlicht wurden und werden. Das generelle Ziel dieses Beitrags ist es deshalb, zu überprüfen, ob und wie die im Rahmen des academic literacies Ansatzes (vgl. Jones et al. 1999; Lea 1999; Lillis 2001) erfolgte erhöhte Aufmerksamkeit für lokale und individuelle Einflussfaktoren auf den wissenschaftlichen Schreibprozess mit dem Konzept lokaler (,kleiner") Kulturen der neueren interkulturellen Schreibforschung kompatibel ist und wie sich diese „kleinen“ kulturellen 
Unterschiede auf textuelle Kohärenzstrukturen auswirken. Im vorgegeben Rahmen kann dieses Ziel allerdings nur beispielhaft anhand qualitativer Analysen erreicht werden.

Dazu wird ein deutschsprachiger wissenschaftlicher Aufsatz, der von einem kompetenten Mitglied einer Diskursgemeinschaft verfasst wurde, mit einem deutschsprachigen wissenschaftlichen Text (Seminararbeit) eines Studierenden verglichen, wobei hauptsächlich die textuellen Makrostrukturen und ausgewählte mikrostrukturelle Eigenschaften im Fokus stehen werden. Die Analyse der funktionalen Phasen der untersuchten Texte erfolgt anhand des bekannten und häufig verwendeten Textsortenmodells von Swales (1990), die Analyse der Makro- und Mikrostrukturen wendet das Kohärenzmodell der rhetorical structure theory (RST, vgl. Mann/Thompson 1988) an (wie die beiden Aspekte „Textsortenstruktur“ und „Kohärenzstruktur“" zusammenhängen, wird in Abschnitt 3 diskutiert).

Das geplante Vorgehen dieses Beitrags basiert auf den folgenden Vorannahmen:

1. Kleine (und große, d. h. nationale) Wissenschaftskulturen wurden und werden häufig auch aufgrund spezifischer Formen der Institutionalisierung von Wissenschaft- und Forschung in einzelnen Kulturkreisen, einzelnen Disziplinen, aber auch einzelnen Forschungsinstitutionen unterschieden (vgl. Holliday 1999). Damit können auch die Texte von Schreibenden, die sich auf unterschiedlichen institutionellen Positionen innerhalb einer Wissenschaftskultur befinden, so betrachtet werden, als wären sie Produkte unterschiedlicher (Sub-)Kulturen und die etwaigen Unterschiede zwischen ihnen können als „kulturelle“ Unterschiede interpretiert werden.

2. Textuelle Kohärenzstrukturen als Realisierungen kommunikativer Intentionen von TextautorInnen variieren mit unterschiedlichen Textsorten (vgl. Gruber/Muntigl 2005; van der Vliet/Redeker 2014; Matthiessen 2014), in einer Textsortenkolonie müssen damit die einzelnen Textsorten bzw. Textsortenvarianten anhand von Unterschieden in der Kohärenzstruktur identifizierbar sein (vgl. dazu etwa Baßler 2003).

In den beiden folgenden Abschnitten werden neuere Kulturkonzeptionen der interkulturellen Rhetorik sowie die RST kurz dargestellt und diskutiert und die Forschungsfragen der Arbeit detailliert dargestellt. Im darauffolgenden empirischen Teil werden zuerst ein „professioneller" und ein studentischer wissenschaftlicher Text in Bezug auf ihre Gesamt(Makro-)struktur miteinander vergleichen. Dann erfolgte eine Mikroanalayse der beiden Texte, die demselben Muster folgt. Im abschließenden Teil werden die Ergebnisse der qualitativen Studie im Lichte der Forschungsfragen diskutiert.

\section{$2 \quad$ Kulturkonzepte in der interkulturellen Schreibforschung}

Im Feld der kulturvergleichenden Schreibforschung, die sich mit sprachlichen und rhetorischen Eigenschaften wissenschaftlicher Aufsätze in verschiedenen Sprachen beschäftigt, ist die sog. ,interkulturelle Rhetorik“ (Connor 2004) als Reaktion auf die seit den 1970er Jahren betriebene kulturvergleichende ('cross-cultural') Rhetorik entstanden, die auf der Basis eines produkt- (d. h. text-) orientierten Ansatzes globale „Sprachkulturen“ miteinander verglichen und die ermittelten Unterschiede anhand einfacher binärer Unterscheidungen (wie leser- vs. schreiberorientierte Kulturen, kollektivistische vs. individualistische Schreibnormen etc.) erklärt hat (vgl. etwa Kaplan 1966; Clyne 1987). 
Im Gegensatz zu den frühen generalisierenden Studien betonen neuere Ansätze die hybride und in sich differenzierte Strukturierung internationaler Wissenschaftsgemeinschaften (vgl. Atkinson 2004; Atkinson/Sohn 2013; Holliday 1999). Dabei wird zwischen sogenannten „großen“ und „kleinen“ Kulturen unterschieden (vgl. Holliday 1999), wobei eine „große Kultur“ dem traditionellen Konzept der ,nationalen“ oder „sprachlichen“ Kultur entspricht, die aber durch viele „kleine“ Kulturen (fachliche, institutionelle, etc.) intern differenziert wird. D. h., dass etwa in einer spezifischen nationalen Forschungsinstitution die „große“ Kultur nur allgemeine grundlegende Faktoren der Wissenschaftsorganisation strukturiert, die durch lokale institutionelle (kleine) Faktoren individuell differenziert und ausgestaltet werden.

Dieser Ansatz ist mit der Konzeption fachlicher Kulturen ('disciplinary cultures') des new rhetoric Ansatzes kompatibel (vgl. etwa Bazerman 1988; Berkenkotter/Huckin 1995) sowie v. a. mit dem sog. academic literacies Ansatz, der die Relevanz lokaler Einflussfaktoren (wie etwa Machtverhältnisse in spezifischen wissenschaftlichen Institutionen, individuelle Schreibbiographien, etc.) auf die wissenschaftliche Textproduktion betont (s. Jones, Turner/Street 1999; Lea 1999; Barton/Hamilton 2000; Clark/Ivanič 1997). Aus der Sicht der VertreterInnen der new rhetoric Studien werden fachliche Kulturen durch das Zusammenspiel disziplinspezifischer Schreibpraktiken und -konventionen mit rhetorischen, epistemologischen Traditionen, sowie fachlichen Methodologien und anderen disziplinspezifischen Praktiken konstituiert. Ein wichtiger Aspekt dabei ist die fachliche Sozialisierung von „NovizInnen“ im Rahmen von sog. „Praxisgemeinschaften“ ('communities of practice', vgl. Berkenkotter/Huckin 1995). Die (wenigen) Studien, die sich bisher mit diesem letzteren Aspekt der wissenschaftlichen Schreibsozialisation beschäftigt haben (für eine rezente Studie mit einer aktuellen Literaturübersicht vgl. Dressen-Hammouda 2008) fanden in allen fachlich relevanten Textaspekten Unterschiede zwischen arrivierten und jungen WissenschaftlerInnen.

Während viele Untersuchungen im Feld des new rhetoric Ansatzes (zumindest implizit) davon ausgehen, dass die fachliche Sozialisation, obwohl sie für den/die Einzelnen schwierig sein mag, fachspezifisch relativ einheitlich verläuft, betont der academic literacies Ansatz den Einfluss lokaler Faktoren (also die Bedeutung der oben erwähnten „kleinen“ Kulturen unterhalb des Niveaus der Einzeldisziplin) für individuelle fachliche Sozialisationsprozesse und die Entwicklung einer disziplinspezifischen Schreibkompetenz (vgl. Ivanič 1998; Lillis 2001).

Darüber hinaus haben rezente Studien zu fachlichen Kulturen auch auf die Bedeutung der lokalen Wissenschaftssprache für die fachliche Sozialisation hingewiesen (vgl. Gnutzmann/Rabe 2014; Hyland 2009, 2013; Mauranen/Perez-Llantada/Swales 2010). Denn obwohl Englisch in vielen Wissenschaftsdisziplinen heute bereits den Status einer Lingua franca für Fachpublikationen hat, erfolgt der größte Teil der universitären und nachuniversitären fachlichen Ausbildung in vielen (europäischen) nicht-englischsprachigen Ländern immer noch in der jeweiligen Landessprache. D. h. während viele Studierende und WissenschaftlerInnen englischsprachige Texte lesen (und ggf. publizieren), so lehren, lernen und forschen sie in einer anderen Sprache. Diese Tatsache hat für die fachliche Sozialisation von Studierenden und JungwissenschaftlerInnen eine nicht zu unterschätzende Bedeutung und prägt auch die lokalen Wissenschaftskulturen in nicht-englischsprachigen Ländern. Auf der lokalen Ebene einzelner Forschungsinstitutionen interagieren damit nationale/sprach- 
spezifische (,große“) Kulturen mit kleineren disziplinspezifischen und noch kleineren institutions- und gruppenspezifischen Kulturen (vgl. Atkinson 2004: 285-286).

Der enge Zusammenhang zwischen fachspezifischen Schreibpraktiken, Textsortenkonventionen (und damit auch der individuellen Textsortenkompetenz) und fachlichen Kulturen sowie deren jeweils lokale, institutions- und gruppenspezifische Ausprägung legen es nahe, dass sich Realisierungen von gleichen oder zumindest ähnlichen Textsorten durch Schreibende, die unterschiedlichen fachlichen (Sub-)kulturen angehören, auch in Bezug auf ihre Kohärenzstruktur unterscheiden, da diese Indikatoren disziplinspezifischer Denk- und Argumentationsmuster darstellen (vgl. Gruber 2007).

\section{Die Rhetorical Structure Theory (RST) als Kohärenzmodell und Methode der Textanalyse}

RST ist eine funktionale Theorie der Textstruktur, die von Mann, Thompson und Matthiessen entwickelt wurde (vgl. Mann/Thompson1986, 1988, 1992; Mann/Matthiessen/Thompson 1992) und die die Beziehungen zwischen Textteilen auf unterschiedlichen Ebenen (von der Ebene der Teilsätze bis zur Ebene von globalen Textabschnitten) beschreibt. Grundsätzlich geht RST davon aus, dass die Beziehungen zwischen Textteilen sprachunabhängig sind, aber sprachspezifisch realisiert werden, wobei es auch einzelsprachlich keine 1:1 Zuordnung zwischen Kohärenzrelationen und sprachlichen Ausdrucksmöglichkeiten gibt. Nach dieser zentralen Modellvorstellung hängen die Beziehungen zwischen Textteilen nicht von Oberflächenmerkmalen des Texts (also Kohäsionsmittel), sondern von der Interpretation eines Texts durch die Rezipierenden ab, damit ist RST ein Modell der Textkohärenz.

RST basiert auf vier grundlegenden Konzepten: (1) Relationen, (2) Schemata, (3) SchemaAnwendungen und (4) Strukturen. Relationen bestehen aus zwei einander nicht überlappenden Textteilen, die gemeinsam eine „Textspanne“ bilden. Das Modell geht davon aus, dass Textspannen einander nicht überlappen, aber selbst wiederum aus Textspannen (einer niedrigeren Textstrukturebene) bestehen können. Die beiden Elemente einer Textspanne sind (üblicherweise) ein „Nukleus“ (N; dieser enthält die ,zentrale“ Information der Textspanne) und ein „Satellit“ (S; dieser enthält Information, die die des Nukleus in irgendeiner Form ergänzt). Der Umfang der Textteile, die eine Textspanne bilden, hängt von der jeweiligen Strukturebene (s. u.) ab, auf der eine Relation die Elemente (Nukleus und Satelliten) einer Textspanne organisiert. Mit dem Teilsatz ('clause') nimmt das Modell allerdings eine untere Grenze für sog. „terminale Knoten“ ('terminal nodes') an, unterhalb derer eine Analyse von Kohärenzrelationen in einem Text nicht mehr als sinnvoll erachtet wird (vgl. Mann/Thompson 1988). Allerdings bedeutet das nicht, dass eine RST- Textanalyse notwendigerweise immer bis zur Ebene des Teilsatzes durchgeführt werden muss, je nach Forschungsfrage können auch die Absätze oder Unterkapitel umfangreicherer Texte die terminalen Knoten einer Analyse darstellen.

Die Definition einer Relation zwischen $\mathrm{N}$ und $\mathrm{S}$ besteht aus vier Komponenten: (1) Bedingungen für den Nukleus, (2) Bedingungen für den Satellit, (3) Bedingungen für die Kombination von Nukleus und Satellit, (4) und aus dem Effekt, den die Verwendung der Relation beim Leser hervorruft. Ein grundlegendes Merkmal des Modells ist, dass diese vier, für jede RST Analyse grundlegenden Komponenten nicht als objektiv gegebene Faktoren 
konzipiert sind, sondern als analytische Leistung der Person, die einen Text analysiert und dabei versucht, die Intentionen des Schreibenden zu modellieren und nach zu vollziehen. Das heißt: im Zuge einer Textanalyse bringt die Analysierende relevante Kontextfaktoren und ihr Wissen über die Autorin sowie über das intendierte Publikum zur Anwendung, um die Relationen, die in einem Text bestehen, zu bestimmen. Eine RST-Analyse ist damit immer auch eine Analyse der (durch den Text nahe gelegten) Intentionen der Autorin bezüglich der Textkohärenz und ihrer Wirkung auf die vorgestellte LeserInnenschaft. RST geht davon aus, dass für die Analyse der Mehrzahl von Texten ein finites Set von Relationen ${ }^{1}$ (das induktiv durch die Analyse von Texten bestimmt wurde) ausreicht, es allerdings notwendig sein kann, für spezifische (fachsprachliche) Textsorten zusätzliche Relationen zu definieren.

Schemata definieren die strukturellen Kombinationsmöglichkeiten von Textteilen und Relationen und damit die in einer RST-Analyse möglichen Textstrukturen. In den Darstellungen des RST-Modells werden üblicherweise fünf verschiedene Arten von Schemata unterscheiden (vgl. Mann/Thompson 1986, 1988), die sich jedoch auf drei Grundkonstellationen reduzieren lassen:

- $\quad$ einfache Nukleus - Satellit Kombinationen: Darunter fällt die Mehrzahl der Relationen. Sie bestehen aus einem Nukleus und einem Satellit, der entweder vor oder nach dem Nukleus stehen kann.

- Schemata mit einem Nukleus und mehreren Satelliten. Ein derartiges Schema kann (1) aus zwei unterschiedlichen Relationen bestehen, die je einen Satellit mit dem Nukleus der Textspanne verbinden; (2) kann in einem derartigen Schema die gleiche Relation zwischen einem Nukleus und mehreren Satelliten herrschen.

- Schemata, in denen mehrere Nuklei ohne Satelliten miteinander kombiniert werden. Hier gibt es zwei Grundmuster: (1) multinukleare Schemata, die prinzipiell nur aus zwei Nuklei bestehen können (z. B. Contrast), oder (2) multinukleare Schemata, die mehr als zwei Nuklei umfassen können (z. B. List).

Textstrukturen ergeben sich in einer RST-Analyse aus der rekurrenten Anwendung von Schemata auf unterschiedlichen Ebenen, wobei die Analyserichtung im Prinzip gleichgültig und auch nicht vorgegeben ist. D.h. eine Analyse kann auf der Ebene der (kleinsten) terminalen Knoten beginnen, diese $\mathrm{zu}$ Textspannen zusammenfassen und diese dann wiederum zu Textspannen der nächsthöheren Ebene kombinieren bis eine hierarchische Struktur entsteht, auf deren höchster Ebene eine Relation den gesamten Text organisiert. Die Analyse kann aber auch genau umgekehrt vorgehen und im ersten Schritt jene Relation identifizieren, die einen Text auf der höchsten Strukturebene organisiert und dann schrittweise die Relationen auf den jeweils nächstniedrigeren Ebenen ermitteln. Beide Analysevarianten sollten, auf denselben Text angewandt, zum selben Ergebnis (d. h. zu einer identischen Textstruktur) führen. RST-Strukturen werden üblicherweise in Diagrammen dargestellt, in denen bogenförmige Pfeile, die die Relationsbezeichnung tragen, die Relationen anzeigen, und horizontale Linien die Textabschnitte symbolisieren, zwischen denen die jeweilige Relation besteht. Senkrechte Linien kennzeichnen den Nukleus einer Textspanne.

1 Eine Auflistung der Relationen des „Standardmodells“ (inklusive Definitionen) findet sich unter www.sfu.ca/rst/01intro/definitions.html. 
Abbildung 1 zeigt ein RST-Diagramm eines Unterabschnitts aus einem der Untersuchungstexte (WP5) aus dem Textkorpus von Gruber et al. (2006). Hier kombinieren die Schreibenden auf der untersten Ebene drei Nuklei, in denen unterschiedliche Konzepte von „Macht“ aus der Sekundärliteratur dargestellt werden, mit einer list-Relation. Auf der nächsthöheren Ebene ,elaborieren“ sie diese Definitionen insofern, als sie spezifische Zusatzinformationen zu einem Aspekt von „Macht“ (nämlich Beeinflussungstaktiken in Paarbeziehungen) bieten.

\begin{tabular}{|c|c|c|c|}
\hline & $\begin{array}{c}1-4 \\
\end{array}$ & \multicolumn{2}{|l|}{ Elaboration } \\
\hline & List & & \multirow{2}{*}{$\begin{array}{l}\text { Hölzl \& Kirchler } \\
\text { (1998) zeigen in ihrer } \\
\text { Untersuchung, dass } \\
\text { sich die } \\
\text { Machtverteilung } \\
\text { zwischen den } \\
\text { Partnern auf die } \\
\text { Beeinflussungstaktiken } \\
\text { auswirken, }(\ldots)\end{array}$} \\
\hline $\begin{array}{l}\text { Wie Radel (1996) in } \\
\text { ihrer Diplomarbeit } \\
\text { anmerkt, wird unter } \\
\text { Dominanz das } \\
\text { Machtverhältnis } \\
\text { zwischen Partnern in } \\
\text { Beziehungen } \\
\text { verstanden. }\end{array}$ & $\begin{array}{l}\text { Macht ist nach } \\
\text { Huston (1983, } \\
\text { zitiert in Radel, } \\
\text { 1996) die } \\
\text { willentliche } \\
\text { Kontrolle, die eine } \\
\text { Person über eine } \\
\text { andere ausübt }\end{array}$ & $\begin{array}{l}\text { und bei Corfman und } \\
\text { Lehman (1987, zitiert } \\
\text { in Radel, 1996) wird } \\
\text { Macht als die } \\
\text { Fähigkeit einer } \\
\text { Person, Einstellungen, } \\
\text { Meinungen oder } \\
\text { Verhalten einer } \\
\text { anderen Person in } \\
\text { beabsichtigter Weise } \\
\text { zu ändern, } \\
\text { bezeichnet. }\end{array}$ & \\
\hline
\end{tabular}

Abbildung 1: RST-Struktur von Abschnitt 2.1, Text WP52

Zwischen der Kohärenzstruktur eines Texts und jenen Strukturmerkmalen, die die Textsorte signalisieren, die der jeweilige Text realisiert, besteht ein systematischer Zusammenhang (vgl. Matthiessen 2014), der allerdings erst für einige Textsorten wie studentische Seminararbeiten (vgl. Gruber/Muntigl 2004) sowie einige persuasive Textsorten (vgl. van der Vliet/Redeker 2014) detaillierter untersucht ist. Grundsätzlich kann man davon ausgehen, dass in den funktionalen Phasen einer spezifischen Textsorte jeweils eine (oder eine relativ begrenzte Zahl von Varianten) typische(r) Kohärenzstruktur(en) realisiert sind und dass auch die lineare Aufeinanderfolge der einzelnen textsortenkonstitutiven Phasen einer Textsorte eine textsortenspezifische hierarchische Kohärenzstruktur konstituiert. Der Mehrwert einer Kombination von Kohärenz- und Textsortenstrukturanalyse eines Texts besteht darin, dass dadurch das Zusammenwirken der textsortenspezifischen illokutiven Struktur eines Texts (die durch die lineare Abfolge seiner textsortenspezifischen Phasen und Züge realisiert wird) mit der hierarchischen Struktur seines internen semantisch-pragmatischen Zusammenhangs (eben seiner Kohärenzstruktur) explizit gemacht werden kann (vgl. zu den Vorteilen einer Mehrebenen-Textanalyse z. B. Fritz 2014).

\footnotetext{
2 Die Texte des studentischen Textkorpus, aus dem auch der im empirischen Teil dieses Aufsatzes analysierte Studierendentext stammt, wurden anonymisiert (s. Gruber et al. 2006). Das Buchstabenkürzel am Beginn jeder Textsigle verweist auf das Subkorpus, aus dem der Text stammt (WP steht für „Wirtschaftspsychologie“). Die Textausschnitte werden im originalen Wortlaut wiedergegeben, Tipp- und Rechtschreibfehler bleiben unmarkiert.
} 
Die Zusammenschau der in Abschnitt 2 diskutierten Resultate der interkulturellen Schreibforschung sowie der in Abschnitt 3 dargestellten Arbeiten zum Zusammenhang zwischen Textsortenmustern und Kohärenzstrukturen deutet darauf hin, dass es entweder kein sprachen- und disziplinübergreifendes Textsortenmuster des wissenschaftlichen Aufsatzes gibt, sondern dass es sich bei Forschungsaufsätzen in unterschiedlichen Sprachen und Disziplinen um eine „Textsortenkolonie“ im Sinne von Bhatia (2004) handelt, die durch verschiedene lokale Textsortenmuster gebildet wird und deren Mitglieder untereinander durch ein System von „Familienähnlichkeiten“ (i.S. Wittgensteins 1971) verbunden sind oder, dass das in den empirischen Wissenschaft häufig realisierte „IMRD“-Schema des wissenschaftliches Aufsatzes in einer überaus großen Zahl von disziplin- und/oder gruppenspezifischen Realisierungsvarianten existiert, dessen prototypisches Zentrum erst zu rekonstruieren wäre.

\section{$4 \quad$ Fragestellungen}

Welche detaillierten Annahmen zur Realisierung von Textsorten durch Schreibende, die unterschiedlichen lokalen Kulturen angehören, lassen sich aus den dargestellten Überlegungen zur interkulturellen Wissenschaftsrhetorik unter einer academic literaciesPerspektive und zu textsortentypischen Kohärenzstrukturen ableiten?

- Neben kulturellen Denk- und Schreibtraditionen im engeren Sinn wirken sich auch institutionelle Unterschiede bzw. die Positionierung von Schreibenden auf unterschiedlichen institutionellen Niveaus auf die Merkmale der Texte aus, die sie verfassen (ob es sich bei diesen Textunterschieden um Realisierungen unterschiedlicher Textsorten oder um Realisierungsvarianten einer Textsorte handelt kann dabei auf der Basis einer qualitativen Studie nicht überprüft werden). Dabei handelt sich sowohl um epistemische wie auch um institutionelle Unterschiede.

- Epistemische Unterschiede resultieren aus:

○ Unterschiedlichem fachlichem Wissen: professionelle Schreibende wissen mehr über das Thema, über das sie schreiben, als Studierende/NovizInnen. ${ }^{3}$

○ Unterschiedlichem Textsortenwissen: professionelle Schreibende sind mit den relevanten Textsorten ihrer Disziplin vertrauter als Studierende/NovizInnen.

- Institutionelle Unterschiede:

- Expertenstatus vs. NovizInnenstatus: Experten schreiben für andere Experten um sie $\mathrm{zu}$ informieren und $\mathrm{zu}$ überzeugen, während NovizInnen schreiben um nachzuweisen, was sie wissen und um die relevanten Textsortenkonventionen zu erwerben.

Aus diesen epistemischen und institutionellen Unterschieden resultieren verschiedene dominante kommunikative Funktionen (und damit auch Kohärenzstrukturen) der Texte, die sie verfassen:

\footnotetext{
${ }^{3}$ Sowohl „ExpertInnen“ wie auch „NovizInnen“ konstituieren Gruppen, die intern wiederum differenziert werden können: die Gruppe der NovizInnen etwa reicht von fortgeschrittenen Studierenden, die ihre erste (BA-) Arbeit verfassen bis zu DoktorandInnen, die ihren ersten Forschungsaufsatz verfassen (müssen).
} 
- Professionelle Schreibende: Primärer Zweck der Textsorte ist die Information einer Diskursgemeinschaft über die eigene Forschung. Wenngleich durch das heute allgemein übliche peer review Verfahren von wissenschaftlichen Texten professionelle Schreibende in einem gewissen Sinn ebenfalls in eine „Prüfungssituation“ gebracht werden, da ihr zur Publikation eingereichter Text überprüft und beurteilt wird, steht bei der ,professionellen“ Textsorte „wissenschaftlicher Aufsatz“ trotzdem die Informations- und Überzeugungsfunktion im Vordergrund.

- Studierende: Primärer Zweck der Textsorte ist das Ablegen einer prüfungsrelevanten Leistung + der Nachweis des Erwerbs von fachlichem Wissen + der Nachweis einer ausreichenden Textsortenkompetenz. Die kommunikative Funktion von Texten Studierender ist insofern beinahe umgekehrt zu der von Texten professioneller WissenschaftlerInnen als Studierende durch ihre Seminararbeiten primär eine beurteilungsrelevante Leistung abgeben um ein (für sie notwendiges) Lehrveranstaltungszeugnis zu erwerben. Das „Einüben“ der Fähigkeit zur wissenschaftlichen Textproduktion ist bei ihnen ein Nebenprodukt dieser Prüfungsleistung. Wissenschaftliche Textsorten, die Studierende im Rahmen ihres universitären Schreibens realisieren, sind damit durch eine spezifische Paradoxie gekennzeichnet, die aus dem simultanen Vorhandensein mehrerer, gleichgewichteter Textsortenzwecke resultiert, durch die der Erwerb einer wissenschaftlichen Textsortenkompetenz im institutionalisierten Lehr-Lernkontext der Universität rekontextualisiert wird.

Gegen die Überlegungen im letzten Punkt könnte man einwenden, dass der Textsortenzweck studentischer (Seminar-) Arbeiten ausschließlich in ihrem Prüfungscharakter liegen könnte und sich damit „professionelle“ wissenschaftlichen Aufsätze und studentische Seminararbeiten auf der Textsortenebene kategorial unterscheiden würden. Dieser Einwand kann aufgrund der Auswertung der Lehrenden- und Studierendeninterviews, die im Rahmen des in Gruber et al. (2006) dargestellten Forschungsprojekts geführt wurden, entkräftet werden. Alle drei Lehrveranstaltungsleiter gaben in den mit ihnen geführten Interviews an, dass für sie das Schreiben einer Seminararbeit auch dem Einüben bzw. dem Erwerb einer wissenschaftlichen Textkompetenz durch die Studierenden diene und damit das Verfassen einer Seminararbeit die erste wissenschaftliche Textproduktion von Studierenden sei $^{4}$.

\section{$5 \quad$ Empirischer Teil}

Der empirische Teil dieses Beitrags beinhaltet zwei große Abschnitte, die ihrerseits jeweils in drei Unterabschnitte gegliedert sind. Im ersten Abschnitt werden die beiden deutschsprachigen Texte mit der idealtypischen Text- Makrostruktur, die die sog. IMRDStruktur (Introduction, Methods, Results, Discussion, vgl. z. B. Swales 1990) vorgibt, sowie untereinander in Bezug auf ihre jeweiligen Makrostrukturen verglichen. Im zweiten Teil werden die Einleitungen der beiden Untersuchungstexte mit den Annahmen zur Phasenstruktur von Einleitungen des Swalesschen Modells sowie miteinander verglichen.

\footnotetext{
4 „Die Seminararbeit ist die kleinste wissenschaftliche Arbeit“ war damals auf der Website des Instituts für Personalwirtschaft der WU Wien zu lesen (Gruber et al. 2006: 31).
} 
Da Textsortenmodelle Textstrukturen lediglich als geordnete Listen aufeinanderfolgender textueller (Sprech-)handlungen konzipieren (vgl. Redeker/Gruber 2014), die die hierarchischen Beziehungen zwischen einzelnen Elementen der textuellen Makro- und Mesostruktur außer Acht lässt (d. h. jene globalen internen Textzusammenhänge, die über die Ebene der unmittelbaren sequenziellen Nachbarschaft hinaus gehen), wird im Sinne einer in letzter Zeit immer wieder geforderten Mehr-Ebenen Textanalyse (vgl. Fritz 2014; Stede 2007) das linguistische Kohärenzmodell der rhetorical structure theory (RST) herangezogen, um die Zusammenhänge zwischen den einzelnen Elementen der untersuchten Textstrukturelemente genauer in den Blick nehmen zu können. Die Grundlagen der RST und ihrer Anwendung bei der Textanalyse wurden oben in Abschnitt 3 dargelegt. Wie in Gruber (2007, 2013) argumentiert, eignet sich die Untersuchung textueller Kohärenzstrukturen auch dafür, ,kulturelle“ Unterschiede zwischen den Texten, die von Angehörigen unterschiedlicher Gruppen verfasst wurden, herauszufinden.

Die beiden deutschsprachigen Texte, die im Folgenden untersucht werden, stammen aus dem Textkorpus, das in Gruber et al. (2006) erhoben und dort auch genauer beschrieben wurde.

\section{Der professionelle Text:}

Nippa, Michael (1997): „Erfolgsfaktoren organisatorischer Veränderungsprozesse in Unternehmen. Ergebnisse einer Expertenbefragung. “ In: Nippa, Michael/Scharfenberg, Heinz (Hrsg): Implementierungsmanagement. Wiesbaden, Gabler: 21-57.

Bei diesem Text handelt es sich um einen Aufsatz aus der Pflichtlektüreliste für das Betriebswirtschaftsseminar „Implementierung im Personalmanagement“, das in der Studie von Gruber et al. (2006) unter anderem untersucht wurde. Die Pflichtlektüre musste bereits vor Semesterbeginn von allen prospektiven SeminarteilnehmerInnen gelesen werden, da über sie, um zum Seminar zugelassen zu werden, vor Seminarbeginn eine Prüfung abzulegen war. Insofern können die Texte der Pflichtlektüre zumindest für den Mikrokontext des untersuchten Seminars als äußerst relevant (und wahrscheinlich in vieler Hinsicht für die Studierenden beispielhaft) angesehen werden. Tatsächlich wurden die Pflichtlektüretexte auch in den Seminararbeiten sehr häufig verwendet und zitiert. Laut Angaben des Lehrveranstaltungsleiters handelte es sich bei allen Texten der Pflichtlektüre um wissenschaftliche Texte nach den Standards des Faches (Personalwirtschaft, PW), da er darauf Wert legte, dass sich die Studierenden an der Wirtschaftsuniversität zumindest während ihres Studiums mit wissenschaftlicher und nicht (wie später in ihrem Berufsleben) mit professioneller Literatur beschäftigen sollten.

\section{Der studentische Text:}

PW 10: „Implementierung von Personalstrategien“

Die Arbeit wurde als eine von zweien in diesem Seminar mit „Sehr gut“ benotet, insgesamt wurden in der Lehrveranstaltung 13 Arbeiten beurteilt. Damit handelt es sich - wieder in Bezug auf den „Mikrokontext“ des untersuchten Seminars - um eine beispielhafte studentische Arbeit. Ein Vergleich der beiden deutschsprachigen Texte gewährleistet damit, dass es sich um einen Vergleich zweier in diesem speziellen Mikrokontext herausragenden Texte handelt, die aber im Rahmen unterschiedlicher institutioneller Rahmenbedingungen 
produziert und rezipiert und von Verfassern auf sehr unterschiedlichen Niveaus der fachlichen Sozialisation verfasst wurden.

\subsection{Die textuellen Makro-Strukturen}

Laut IMRD-Modell (soweit es sich tatsächlich um ein „Modell“ und nicht um eine schlichte Faustregel für den Textaufbau handelt) wird in der Einleitung eines Forschungsaufsatzes eine Forschungsfrage entwickelt, die im Rest des Texts beantwortet wird (der Text realisiert also eine „Problem-Lösung“-Struktur, vgl. die solutionhood-Relation ${ }^{5}$ auf der höchsten RSTStrukturebene in Abb. 1). Der „Methoden“-Abschnitt (in dem auch die Datenerhebung beschrieben wird) gibt dabei den Rahmen vor, innerhalb dessen die Resultate und deren Interpretation vom Leser überhaupt erst korrekt verarbeitet bzw. verstanden werden kann (vgl. die circumstance-Relation auf der 2. Ebene in Abb. 1), der „Diskussion“-Abschnitt interpretiert schließlich die Resultate (vgl. Ebene 3 in Abb. 1). Aus dieser Kurzbeschreibung der internen rhetorischen Zusammenhänge der einzelnen Teile der IMRD-Struktur lässt sich folgende (theoretische) Makro-Kohärenzstruktur eines empirischen wissenschaftlichen Aufsatzes annehmen:

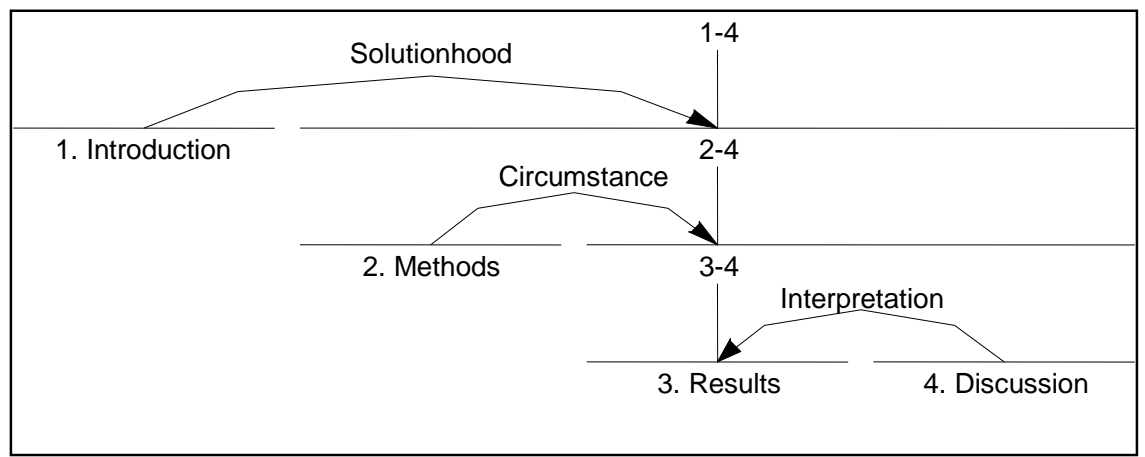

Abbildung 2: RST Struktur eines wissenschaftlichen Aufsatzes, der die IMRD Struktur realisiert

Die Kohärenzstruktur in Abb. 1 zeigt, dass die Kohärenzstruktur eines IMRD-Texts typischerweise einen zentralen Nukleusbereich hat (vgl. den Bereich, der durch die senkrechten Linien, die den Nukleus der jeweiligen Strukturebene signalisieren, markiert ist), der den Resultate- Abschnitt als einzigen terminalen Knoten beinhaltet. Auf diesen zentralen Bereich beziehen sich prospektiv alle vorher kommenden und retrospektiv alle nachher folgenden Textteile (vgl. die Pfeilrichtungen, die von den jeweiligen terminalen Satelliten zum Nukleusbereich führen). Damit bildet die Kohärenzstruktur eines Texts, der die IMRD Struktur realisiert, fast prototypisch eine epistemische Orientierung ab, die einen Schwerpunkt auf die Lösung eines vorgegebenen Problems und deren empirischer Überprüfung bzw. Absicherung legt. Dieser Denk- und Schreibstil wurde bereits von Galtung als der „saxonische“ intellektuelle Stil identifiziert (vgl. Galtung 1985) und wird auch heute noch als prototypisch anglo-amerikanische Tradition des primär problemlösenden Texts den kontinentaleuropäischen „Denk“-Texten ('think-texts') gegenüber gestellt, in denen es primär darum gehe, eine Vielzahl von Ansätzen einander gegenüber zu stellen und gegeneinander abzuwägen (vgl. Rienecker/Stray Jörgensen 2003).

\footnotetext{
${ }^{5}$ Die Relationsdefinitionen sind vielfach aufgrund der Relationsnamen weitgehend selbsterklärend, trotzdem werden im Anhang die in dieser Arbeit verwendeten Relationen und ihre Definitionen (basierend auf der RSTWebsite, www.sfu.ca/rst/01intro/definitions.html) aufgelistet.
} 
Nach dieser kurzen Darstellung des IMRD Modells können wir uns der Analyse der beiden konkreten deutschsprachigen Texte zuwenden, um Übereinstimmungen und Unterschiede zu identifizieren.

\subsubsection{Der professionelle Text}

Bei den beiden deutschsprachigen Texten ist es sinnvoll, vor der Darstellung und Diskussion ihrer Kohärenzstruktur, kurz auf ihre inhaltliche Gliederung einzugehen, da (zumindest bei wissenschaftlichen Texten) Inhaltsgliederung und Kohärenzstruktur häufig insofern zusammenhängen als die Kapitel und Unterkapitel der inhaltlichen Struktur meist die terminalen Knoten (Textspannen) der Makrokohärenzstruktur bilden (vgl. Gruber 2014).

Der professionelle Text hat folgende inhaltliche Gliederung:

- (Einleitung)

- Konzeption und empirische Basis

- Gesamtergebnisse

○ Definition und Erfolgsmaßstäbe organisatorischer Veränderungsprozesse in der Praxis

○ Unterschiede zwischen Konzeptions- und Implementierungsprojekten

○ Barrieren und Motoren organisatorischer Veränderungsprozesse in der Praxis

○ Einschätzungen zum Erfolg von Umsetzungsprojekten und Managementkonzepten

- Ausgewählte Merkmale eines erfolgreichen Implementierungsmanagements

○ „System change“ oder „culture change“"?

- Erfolgsgeheimnisse der Praxis?

\section{Abbildung 3: Inhaltlicher Aufbau des professionellen Texts}

In der Darstellung oben (Abb. 2) ist der Abschnitt „Einleitung“ deshalb in Klammern gesetzt, da er im Text nicht durch eine eigene Überschrift gekennzeichnet ist, sondern direkt auf den Aufsatztitel folgt. Darüber hinaus fällt an diesem Text auf, dass seine (hierarchische und sequentielle) Struktur nicht durch die sonst in Wissenschaftstexten übliche Dezimalnummerierung signalisiert wird.

Auf der Makroebene ergibt sich nach einer genaueren Analyse die folgende Kohärenzstruktur des Texts (Abb. 4): 


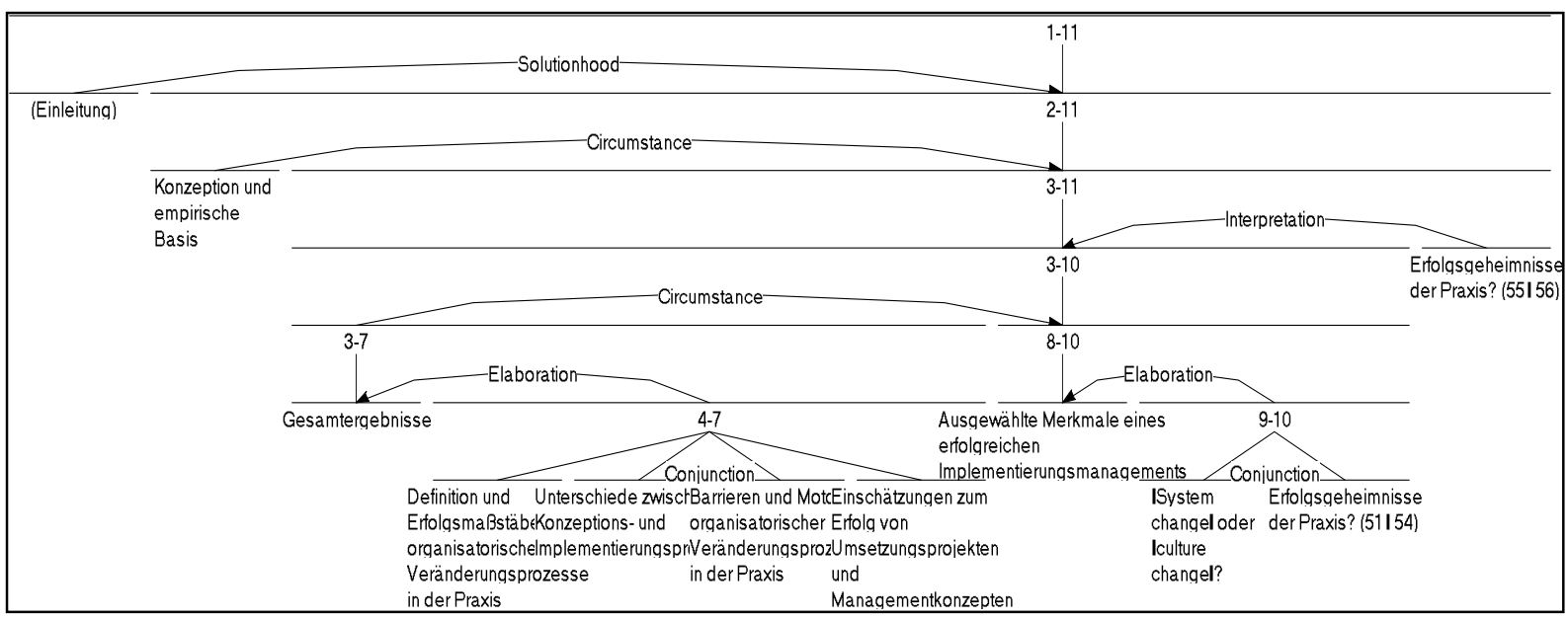

Abbildung 4: RST Struktur des professionellen Texts

Folgende Merkmale der Kohärenzstruktur des Gesamttexts sind im Zusammenhang dieser Untersuchung relevant: zwischen der Einleitung (die allerdings nicht als solche gekennzeichnet ist) und dem Rest des Texts wird vom Autor eine solutionhood- (d. h. eine Problemlösungs-) Relation signalisiert, zwischen dem 2. Abschnitt („Konzeption und empirische Basis") und dem Rest des Texts wird eine circumstance-Relation etabliert (d. h. es wird der konzeptuelle Rahmen und die empirische Basis der Untersuchung präsentiert, die es den Lesenden ermöglichen, die Resultate sinnvoll in den Gesamtzusammenhang der Untersuchung und des Texts einzuordnen) und zwischen den letzten beiden Seiten des Texts (die allerdings keinen eigens gekennzeichneten Textabschnitt bilden) und den vorhergehenden Teilen der beiden Resultate-Kapitel besteht eine interpretation-Relation. Damit findet sich auf den höchsten Ebenen der Makrostruktur eine Kohärenzstruktur, die dem IMRD Schema entspricht (vgl. Abb. 1), durch die inhaltliche und formale Gliederung allerdings nur teilweise signalisiert wird. Darüber hinaus ist der Resultate-Teil in zwei große Teile gegliedert, die eine relativ komplexe interne Struktur aufweisen. Die beiden großen Resultate-Kapitel haben dabei Hauptüberschriften, unter denen sich kein Text befindet, vielmehr beginnt unter jeder Hauptüberschrift sofort ein Unterkapitel (es handelt sich um sog. „leere Überschriften“ wie sie auch häufig in studentischen Arbeiten vorkommen, vgl. Gruber 2014). Die elaboration-Relation zwischen den Haupt- und den folgenden Unterüberschriften des Resultate-Kapitels (also die Tatsache, dass in den einzelnen Unterkapiteln einzelne Aspekte ausgearbeitet werden, die durch die Hauptüberschriften angekündigt werden) wird nicht explizit signalisiert, sondern muss inhaltlich erschlossen werden.

Die Abwesenheit expliziter Textstrukturhinweise ist typisch für den gesamten Text. Die Kohärenzstruktur wird kaum durch textstrukturierende Metakommunikation signalisiert. Es gibt auf 36 Textseiten nur fünf textstrukturierende metakommunikative Sequenzen, von denen sich vier ausschließlich auf die lokale Textorganisation (d. h. auf den Folgesatz oder auf einige vorhergehende Sätze) beziehen (d. h. 0,14 metakommunikative Sequenzen/Textseite), nur auf S. 51 findet sich (unmittelbar unter der Überschrift „Erfolgsgeheimnisse der Praxis?“) eine textstrukturierender metakommunikativer Absatz, der den abschließenden Teil des Resultate-Kapitels vorstrukturiert. 


\subsubsection{Der studentische Text (PW10)}

Vor einer eingehenderen Diskussion der Textmakrostruktur des studentischen Texts muss betont werden, dass in der Aufgabenstellung zum Seminar (in einem zu Semesterbeginn im Seminar ausgeteilten Handout sowie auf der zum Untersuchungszeitraum aktuellen Institutshomepage, vgl. Gruber et al. 2006) einerseits auf die Wichtigkeit der Entwicklung einer eigenständigen Fragestellung in der Arbeit (s. unten die Struktur der Einleitung) sowie auf die Notwendigkeit eines „roten Fadens“ (d. h. der Notwendigkeit einer erkennbaren Kohärenzstruktur), der sich durch die Arbeit ziehe, hingewiesen wurde.

Der studentische Text weist folgende inhaltliche Gliederung auf:

\section{Problemstellung}

2. Definition, Abgrenzung und Beschreibung der Begriffe

2.1. Implementierung

2.1.1. Definition, Abgrenzung Entwicklung der Bedeutung

2.1.2. Die wichtigsten Beeinflussungsfaktoren einer Implementierung

2.1.3. Auslöser einer Implementierung

2.1.4. Implementierungsziel und Implementierungserfolg

2.1.5. Organisationsform der Implementierung

2.1.6. Implementierungsstrategien, -stile, -ansätze

2.1.7. Unterstützende Faktoren bei der Implementierung

\subsection{Personalstrategien}

2.2.1. Begriff, Definition, Abgrenzung

2.2.2. Unternehmensstrategie und Personalstrategie

2.2.3. Initiatoren und Auslöser einer Personalstrategie

2.2.4. Träger und Bezugsgruppen

2.2.5. Beeinflussende Faktoren, Handlungsrahmen, Umfeld

\section{Implementierung von Personalstrategien}

3.1. Einleitung

3.2. Stand der Forschung

3.3. Organisationsform

3.4. Barrieren und Akzeptanz

3.5. Top-Management Support

\section{Conclusio}

\section{Abbildung 5: Inhaltlicher Aufbau des studentischen Texts}

Auffällig an dieser Inhaltsgliederung ist einerseits der relativ symmetrische Aufbau der beiden (umfangreichen) Unterkapitel von Kapitel 2, in denen die beiden zentralen Begriffe „Implementierung“ und „Personalstrategie“ genauer dargestellt werden, sowie die Überschrift der Einleitung mit „Problemstellung“. Letzteres ist wahrscheinlich darauf zurückzuführen, dass die Notwendigkeit der Entwicklung einer eigenständigen Fragestellung in den Seminarunterlagen (und auch im Seminar selbst) mehrfach betont wurden. Ersteres (die parallele Gliederung der beiden Unterkapitel von Kapitel 2) erinnert stark an die von 
Clyne (1987) beschriebene symmetrische Textstrukturierung englischsprachiger Aufsätze und wäre damit für einen deutschsprachigen studentischen Text nicht unbedingt zu erwarten ${ }^{6}$.

Die Makrokohärenzstruktur des studentischen Texts ist in Abb. 6 dargestellt:

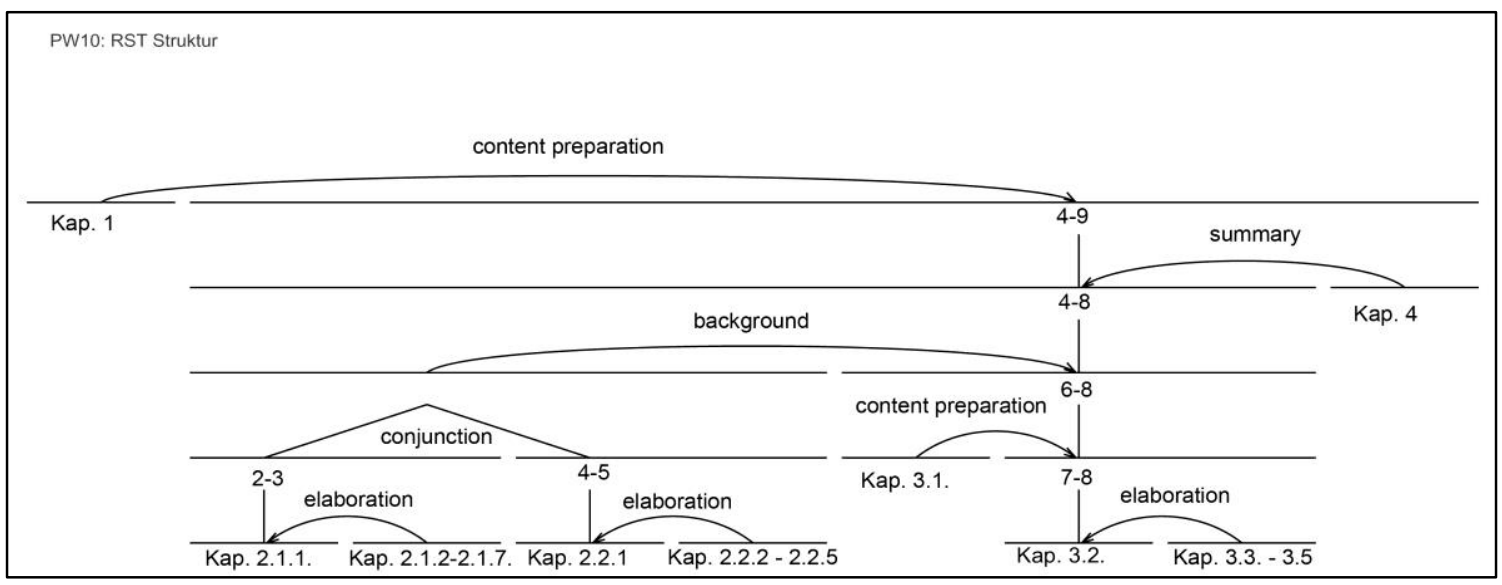

Abbildung 6: RST Struktur des studentischen Texts

Die höchsten drei Ebenen der Kohärenzstruktur des studentischen Texts scheinen auf den ersten Blick parallel zum professionellen Text (und dem IMRD-Schema) strukturiert zu sein (es handelt sich zwar um eine theoretische Arbeit, allerdings wird eine ProblemLösungsstruktur signalisiert), wieder gibt es einen zentralen (komplex strukturierten) Nukleusbereich, auf den die vorhergehenden Textteile vorverweisen und auf den der letzte Teil zurückverweist. Allerdings verwendet der studentische Autor andere Kohärenzrelationen als der professionelle Schreiber. Darüber hinaus ist im studentischen Text kein Methodenteil vorhanden, weil es sich um eine reine Literaturübersichtsarbeit handelt.

Worin bestehen aber auf dieser globalen Ebene die Charakteristika des studentischen Texts und seine Unterschiede zum professionellen Text? Content preparation und summary sind im Vergleich zu solutionhood und interpretation insofern „schwächere“ Kohärenzrelationen als sie in den Lesern keine kognitiven Effekte auslösen (z. B. indem sie ihm/ihr einen Wissensoder Erkenntnisgewinn vermitteln, indem eben ein Problem gelöst ('solutionhood') oder vorher präsentierte Inhalte in einen theoretischen Rahmen eingebunden werden (interpretation); zum Zusammenhang zwischen Kohärenzstrukturen und kognitiven Prozessen vgl. Sanders/Schilperood 2008), sondern sie orientieren die Leser prospektiv auf bestimmte Aspekte des Folgetexts (content preparation) bzw. fassen bestimmte Aspekte retrospektiv nochmals zusammen (summary). Man kann also sagen, dass der studentische Text (im Gegensatz zum professionellen Text) auf der Makroebene kaum argumentativ strukturiert ist. Außerdem suggeriert der studentische Text durch seine Kapitelüberschriften in den Kapiteln 1 und 4 eine andere Struktur als er tatsächlich hat: „Problemstellung“ und

6 Obwohl Clyne's Studie zu Recht aufgrund methodischer Schwächen und wegen ihres unleugbaren Anglozentrismus kritisiert wurde (vgl. etwa Gnutzmann/Lange 1990), wurden einige der diskursiven Phänomene (wie eben der von ihm postulierte parallele Textaufbau englischsprachiger Texte) in den wenigen späteren Vergleichen des deutsch- und englischsprachigen Wissenschaftsstils nicht mehr untersucht. Gleichzeitig zeigt ein Blick auf die Liste jener Textmerkmale, die Rienecker/Stray Jörgensen (2003: 105) als typisch für das „kontinentale Schreiben“ auflisten, eine verblüffende Ähnlichkeit mit jenen Textaspekten, die Clyne in seiner Studie als Unterscheidungskriterien zwischen deutschsprachigen und englischsprachigen wissenschaftlichen Aufsätzen anführt. 
„Conclusio“ scheinen eine argumentative Struktur anzukündigen, die der des professionellen Texts (mit der erwähnten Ausnahme, dass es keinen Methodenteil gibt) bzw. eines an die IMRD Struktur angelehnten Problem-Lösungsschemas entspricht, allerdings wird diese Erwartung durch den Inhalt der beiden Kapitel nicht erfüllt, denn durch eine inhaltliche Vorbereitung ('content-preparation') wird der/die Lesende lediglich auf den Inhalt des Folgetexts vorbereitet (ohne dass tatsächlich ein Forschungs-,,Problem“ formuliert werden würde) und eine Zusammenfassung ('summary') präsentiert nur eine Kurzfassung aber keine abschließende theoretische Einordung der zuvor dargestellten Inhalte.

Auch im studentischen Text sind explizite metakommunikative textstrukturierende Verweise eher selten, sie kommen aber (relativ gesehen) doppelt so häufig vor wie im professionellen Text: es gibt auf 16 Textseiten fünf explizite (und zum Teil absatzlange) metakommunikative Sequenzen (0,31 metakommunikative Sequenzen/Textseite), die sich immer auf umfangreichere Teile des Texts (oder auch auf den Gesamttext) beziehen. Im studentischen Text wird die Kohärenzstruktur darüber hinaus auch durch die Unterkapitelnummerierung signalisiert bzw. gestützt, d. h. den LeserInnen wird durch die Dezimalnummerierung signalisiert, welche Textteile auf derselben Strukturebene liegen und welche untergeordnet sind. Das ist im professionellen Text nicht der Fall, in dem es keine Kapitelnummerierung gibt, und in dem weder die Einleitung eine Überschrift trägt noch der interpretation - Satellit am Textende durch eine Kapitel - oder Unterkapitelüberschrift markiert sind. Insofern signalisiert der studentische Text seine Kohärenzstruktur viel expliziter als der professionelle Text.

\subsection{Die textuellen Meso- und Mikrostrukturen}

Die interne Struktur einer Einleitung besteht im Swalesschen Modell aus drei Phasen, die wiederum typischerweise durch jeweils mehrere „Züge“ ('moves') realisiert werden. Diese sind in ihrer strikten Abfolge, die das ursprüngliche Modell (vgl. Swales 1990) annimmt, in der folgenden Auflistung dargestellt. Die spätere Modellformulierung (vgl. Swales 2004) sieht allerdings durchaus die Möglichkeit der zyklischen Wiederholbarkeit einzelner Phasen vor:

- Etablieren eines Territoriums:

- Die Wichtigkeit des Themas behaupten

- Verallgemeinerungen über das Thema machen

○ Einen Forschungsüberblick geben

- Etablieren einer Nische:

- Eine Gegenbehauptung gegen eine bestehende Annahme aufstellen

○ Aufzeigen einer Forschungslücke

- Eine Frage oder ein Problem formulieren

○ Eine Forschungstradition fortführen

- Besetzen der Nische:

○ Skizzieren des Zwecks der eigenen Arbeit

○ Ankündigung der wichtigsten Merkmale der eigenen Arbeit

- Ankündigung der wichtigsten Resultate

- Skizzieren des Aufbaus der Arbeit 
Aufgrund der in der aktuellen Modellversion möglichen Zyklizität der Phasen und da die einzelnen Züge innerhalb der Phasen sowohl alternativ (v.a. in Phase 2) wie auch kumulativ (v. a. in den beiden anderen Phasen) realisiert werden können, ist es nicht möglich eine (einzige) theoretische Kohärenzstruktur einer Einleitung, die dem Swalesschen Modell entspricht, vorzuschlagen, da die Kombinationsmöglichkeiten von Zügen innerhalb und zwischen den Phasen viel zu groß sind. Klar scheint zu sein, dass eine Einleitung, die dem Modell entspricht, keinen zentralen Nukleusbereich aufweisen wird, da die drei Phasen schrittweise aufeinander bezogen sind, wobei jede folgende Phase einen Teilaspekt der vorhergehenden Phase aufnimmt und ihn weiter entwickelt. Darüber hinaus ist der letzte Zug in Phase drei (Skizzieren des Aufbaus der Arbeit) nicht auf den Rest der Einleitung, sondern prospektiv auf den Folgetext bezogen, wodurch im gesamten IMRD Schema (s.o. Abb. 1) eigentlich zwischen den beiden höchsten Hierarchieebenen eine „Zwischenebene“ eingezogen wird, in der der Satellit einer preparation-Relation auf den gesamten Folgetext als Nukleus verweist.

Obwohl Swales' Textsortenuntersuchungen des wissenschaftlichen Aufsatzes eigentlich auf der Analyse englischsprachiger Texte aus dem Bereich der Naturwissenschaft beruhte, wurde gerade die Phasen- und Zugstruktur der Einleitung als Basis vieler kulturvergleichender Studien herangezogen, in denen auch Texte anderer Disziplinen untersucht wurden (z. B. Gnutzmann/Lange 1990; Taylor/Chen 1991; Loi 2010). Diese Vergleichsstudien zeigen häufig, dass in nicht-englischsprachigen Einleitungen dieselben rhetorischen Phasen und Züge wie in englischsprachigen Einleitungen $\mathrm{zu}$ finden sind, allerdings in je unterschiedlichen Anordnungen (d. h. Reihenfolgen).

Darüber hinaus scheint das Swalessche Phasenmodell der Einleitung zumindest implizit auch viele deutschsprachige Schreibratgeber für Studierende der Sozialwissenschaften beeinflusst zu haben, wenn es etwa in einer Einführung ins wissenschaftliche Arbeiten für Studierende der Sozial- und Wirtschaftswissenschaften heißt: „Die Einleitung hat folgende Funktionen:

- Weckung des Interesses des Lesers an der Arbeit

- Hinführung des Lesers zum Thema

- Darlegung der Problemstellung der Arbeit

- Darstellung der Struktur der Arbeit“" (Ebster/Stalzer 2003: 82)

Auf der Basis dieser Tatsachen lässt sich das Swalessche Modell der Einleitung des wissenschaftlichen Aufsatzes auch als Grundlage eines Vergleichs nicht-englischer Einleitungen von Textsortenvarianten dieser Textsorte heranziehen.

\subsubsection{Die Kohärenzstruktur der Einleitung des professionellen Texts}

Die Einleitung des professionellen Texts umfasst die lineare Abfolge folgender rhetorischer Züge (Sprechhandlungen) - ihre Benennungen zeigen bereits, dass die Phasen und Züge, die das Swalesschen Modell anbietet, nur teilweise ausreichen, um die rhetorischen Funktionen, die in dieser Einleitung auf der textuellen Mesoebene realisiert werden, zu beschreiben:

- Verallgemeinerung über das Thema (1. Absatz, gleichzeitig: Formulierung eines Problems)

- Illustration dieser Verallgemeinerung (2. Absatz)

- Anführen (zweier) möglicher Gründe für das Problem (3. Absatz, Z1-5; Z5-8)

ISSN 1615-3014 
- Gegenbehauptung gegen diese Gründe (4. Absatz)

- Reformulieren des (Insistieren auf dem) Problem (5. Absatz =1 Satz)

- Anführen von Belegen (Literaturverweise) für das Bestehen des Problems (6. Absatz)

- Skizzieren des Zwecks der eigenen Arbeit, Auflistung der Forschungsfragen (7. Absatz)

Daraus ergibt sich folgende Kohärenzstruktur der Einleitung:

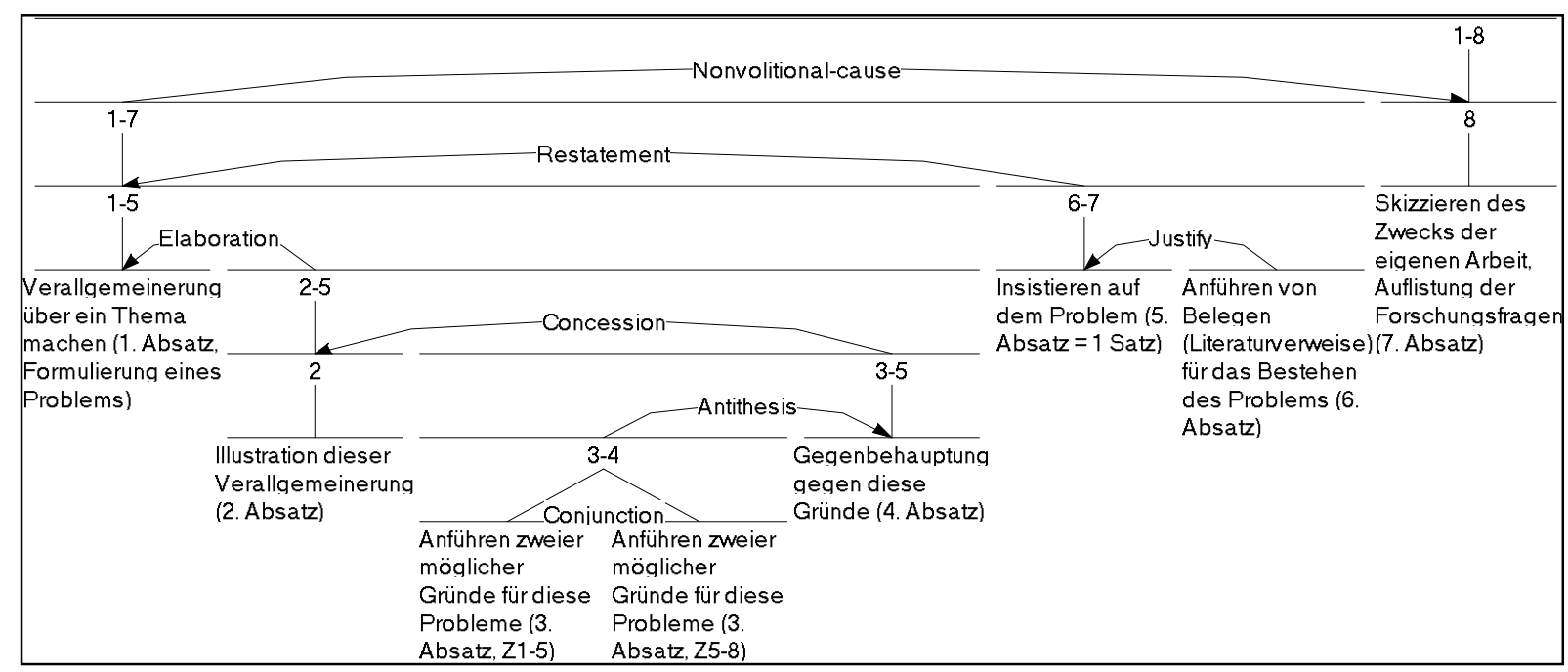

Abbildung 7: RST Struktur der Einleitung des professionellen Texts

Die Kohärenzstruktur der Einleitung des Texts weist folgende Charakteristika auf: Die „Verallgemeinerung über das Thema/Formulierung des Problems“ (Realisierung der Phase 1 „Etablieren des Territoriums“ im Swalesschen Modell) dient gleichzeitig dazu, auch das zentrale Problem, das in der Arbeit bearbeitet werden soll, zu formulieren. Damit werden die Phasen 1 und 2 des Swalesschen Modells simultan am Textbeginn realisiert. Der größte Teil der restlichen Einleitung dient dann dazu, die Problemstellung zu rechtfertigen, was in einer relativ komplexen Argumentationsstruktur resultiert (vgl. die concession, antithesis und justify-Relationen auf den Textebenen 3, 4 und 5, Abb. 7), an deren Ende die Problemstellung nochmals wiederholt wird (vgl. die restatement-Relation auf Textebene 2 in Abb. 7). Das Skizzieren des Zwecks der eigenen Arbeit erfolgt am Ende der Einleitung relativ unvermittelt, die Kausalbeziehung (vgl. die non-volitional cause-Relation auf Textebene 1, Abb. 7)) zwischen dem komplex argumentierten größten Teil der Einleitung, in dem die Verallgemeinerung über das Thema/Formulierung des Problems sowohl argumentativ gestützt wie auch anhand von Literaturverweisen gerechtfertigt wird, und der Ankündigung der Details der Untersuchung am Ende der Einleitung muss von den LeserInnen weitgehend selbst erschlossen werden.

Der Grund dafür ist ein ähnlicher wie in der Makrostruktur des Gesamttexts: die Kohärenzrelationen in der Einleitung werden kaum explizit sprachlich signalisiert, obwohl auf der Mesoebene des Texts durchaus auch argumentative Relationen (antithesis, concession, justify) vorkommen, die im Vergleich zu nicht argumentativen Relationen nicht nur im Deutschen häufig sprachlich markiert werden (vgl. van der Vliet/Redeker 2014; Speyer/Fetzer 2014). Der letzte „Zug“ einer am Swalesschen Modell orientierten Einleitung (die Darstellung der Struktur der Arbeit) fehlt überhaupt - durchaus im Einklang mit den auch oben erwähnten weitgehend fehlenden metakommunikativen textstrukturierenden Sequenzen. 


\subsubsection{Die Kohärenzstruktur der Einleitung des studentischen Texts}

Abgesehen von den verschiedenen Kohärenzstrukturen fällt die stark unterschiedliche Länge der beiden Einleitungen auf: die ,professionelle“ Einleitung umfasst zwei Druckseiten, während die studentische Einleitung mit nur einer halben Seite auskommt. Die Einleitung des studentischen Texts realisiert folgende Phasen bzw. Züge des Swaleschen Modells ${ }^{7}$ :

- Besetzen der Nische: Skizzieren des Zwecks der eigenen Arbeit

- Etablieren einer Nische: Formulierung eines Problems

- Besetzen der Nische: Ankündigung der wichtigsten Merkmale der eigenen Arbeit

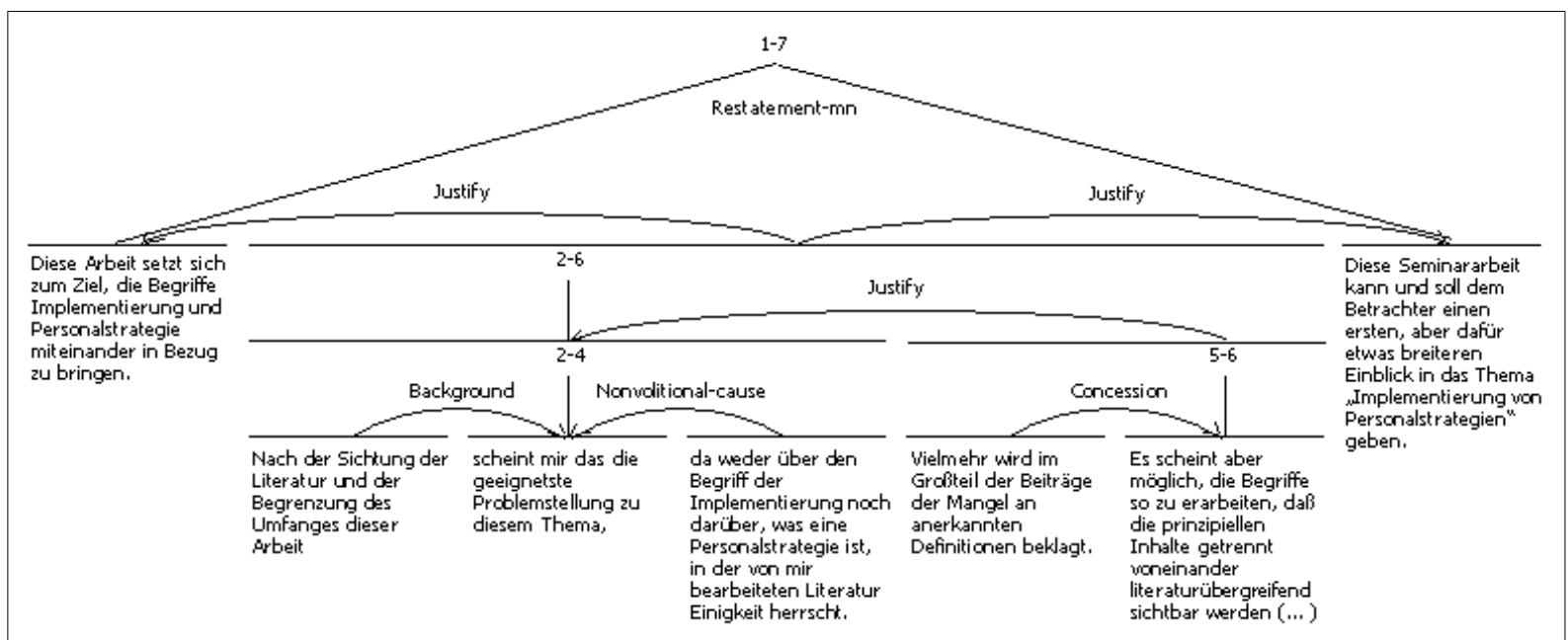

Abbildung 8: RST Struktur der Einleitung des studentischen Texts

Der studentische Text weist damit ausschließlich Phasen bzw. Züge auf, die das Swalessche Modell vorsieht, allerdings wird eine Nische etabliert und besetzt, die gleichzeitig als das „Territorium“, innerhalb dessen sich der Text positioniert, präsentiert wird. Denn der Forschungsüberblick (durch den eigentlich das Territorium etabliert werden sollte) wird nicht in der Einleitung präsentiert, sondern er wird als Ziel für den Folgetext definiert. Das kann mit der primären Funktion des studentischen Texts erklärt werden: der Studierende will dem Seminarleiter zeigen, dass er die relevante Literatur zu seinem Thema recherchiert und aufgearbeitet hat, er will eigentlich keine Forschungslücke schließen. Damit tritt der Prüfungsaspekt der Arbeit in den Vordergrund.

Darüber hinaus weckt die Überschrift der Einleitung eine inhaltliche Erwartung, die der Text selbst nicht erfüllt (s.o. Abschnitt 5.1.2.). Denn die Einleitung formuliert keine „Problemstellung“, sondern bereitet den/die LeserIn lediglich auf den inhaltlichen Hauptteil des Texts vor (vgl. die Relation content preparation auf der ersten Textstrukturebene in Abb. 6). Wie im professionellen Text wird zwar die Textintention (aber eben keine echte Problembzw. Fragestellung) einleitend formuliert und abschließend nochmals wiederholt (vgl. die restatement $m n$ Relation auf der obersten hierarchischen Textebene in Abb. 8). Ebenfalls wie

\footnotetext{
${ }^{7}$ Obwohl es sich bei der Seminararbeit um keinen wissenschaftlichen Aufsatz im engen Sinne handelt (sondern eher um die Simulation eines solchen, vgl. oben die Zusammenfassung der Interviewaussagen der Seminarleiter) werden im studentischen Text eher Phasen und Züge, die dem Swalesschen Modell, realisiert als im professionellen Text.
} 
im professionellen Text wird die (erste Formulierung der) Textintention argumentativ gestützt (vgl. die justify, non-volitional cause- und concession-Relationen auf den Textebenen 2 und 3 in Abb. 8). Allerdings dient die Formulierung der Textintention im studentischen Text nicht dazu, eine echte Fragestellung argumentativ zu stützen, so wie das im professionellen Text der Fall ist, sondern sie wird am Anfang der Einleitung präsentiert und an ihrem Ende wiederholt. Daraus folgt auch die (im RST Modell eigentlich nicht vorgesehene) auffällige Schemastruktur auf der 2. Textstrukturebene, in der ein Satellit (nämlich der mittlere Teil der Einleitung) durch eine justify-Relation mit 2 Nuklei (den beiden Abschnitten, in denen die Textintention am Anfang und am Ende der Einleitung präsentiert wird) verbunden ist. All das kann als Hinweis auf den Prüfungscharakter der Arbeit gewertet werden, allerdings in anderer Weise als die Tatsache, dass in der Arbeit ausschließlich Literatur aufgearbeitet wurde: der studentische Autor zeigt damit, dass er sich an die Vorgaben des Lehrveranstaltungsleiters bzw. der Institutswebsite hält, in denen explizit die Formulierung einer „Fragestellung“ als Ausgangspunkt für eine Seminararbeit gefordert wird.

In beiden Texten fehlt eine explizite structural preparation, d. h. eine prospektive Darstellung des inhaltlichen Textaufbaus, die studentische Einleitung dient jedoch in ihrer Gesamtheit dazu, den Text inhaltlich vorzubereiten (d. h. darzustellen, was der Autor im Folgenden tun wird). Während aber im professionellen Text die Reformulierung der Problemstellung viel kürzer als ihre erste Darstellung ist und eigentlich dazu dient, zu den Zielen der eigenen Arbeit hinzuführen, sind die beiden Formulierungen im studentischen Text praktisch gleich lang und wirken deshalb wie Paraphrasen ein und desselben Gedankens.

Ähnlich wie der Vergleich der beiden Textmakrostrukturen zeigt auch der Vergleich der Einleitungen des professionellen und studentischen Texts oberflächliche Parallelen in Bezug auf die realisierten Phasen, aber relativ gravierende Unterschiede in Bezug auf die Kohärenzrelationen, die zwischen diesen Phasen bestehen. Darüber hinaus weist der professionelle Text in seiner Einleitung eine Reihe von Phasen und Zügen auf, die im Swalessche Modell nicht vorgesehen sind, obwohl diese in vielen sozialwissenschaftlichen Disziplinen und nicht-englischen Wissenschaftsgemeinschaften in Einleitungen von Forschungsaufsätzen gefunden wurden. Das zeigt, dass es (zumindest in Einzelfällen) auch in professionellen Texten zu merkbaren Abweichungen von diesem Textsortenmodell kommen kann.

\section{Diskussion der Ergebnisse}

Der Vergleich der Makrostrukturen der beiden Texte zeigt, dass das IMRD - Schema insofern eine Relevanz für (das Verfassen und) die Analyse von deutschsprachigen wissenschaftlichen Texten hat, als es eine dreigliedrige Grobstruktur der Texte nahelegt, in der ein zentraler (nuklearer) Mittelteil durch ein oder zwei vorbereitende Textteile eingeleitet und im Rahmen eines abschließenden Teils resümiert bzw. interpretiert wird. Allerdings zeigen sich zwischen dem professionellen und dem studentischen Text insofern Unterschiede als im ersteren die Kohärenzrelationen zwischen diesen drei Teilen einer Problemlösungsstruktur (Formulierung des Problems - Ausarbeitung/Präsentation einer Lösung - Bewertung/Interpretation dieser Lösung) entsprechen, während im studentischen Text die Relationen eher beschreibend sind (Vorbereitung auf den Folgetext am Beginn, Zusammenfassung des Mittelteils am Ende), 
obwohl die Kapitelüberschriften eine ähnliche Problemlösungsstruktur wie im professionellen Text signalisieren.

Diese Diskrepanz lässt sich durch die unterschiedlichen institutionellen Positionen der Autoren und den daraus resultierenden verschiedenen dominanten Textfunktionen erklären: der professionelle Text hat die explizite Funktion, ein Fachpublikum über die Ergebnisse einer vom Autor durchgeführten Studie zu informieren. Diese Studie bezog sich in einigen Aspekten auf offene Probleme der Forschung. Im Gegensatz dazu bestand für den studentischen Text zwar die explizite Anforderung im Text eine Problemstellung zu formulieren und dann zu bearbeiten, de facto präsentiert der Text aber eine Literaturübersicht zu einem vorgegebenen Thema und setzt die Standpunkte und Ergebnisse einzelner Untersuchungen zueinander in Beziehung. Damit tritt im Text der Prüfungsaspekt der Arbeit gegenüber dem problemlösenden (potentiell neues Wissen generierenden) Gesichtspunkt in den Vordergrund. Man könnte nun argumentieren, dass es sich aufgrund dieser unterschiedlichen dominanten Textfunktionen bei den beiden Texten um die Realisierung unterschiedlicher Textsorten handelt. Dieser Sichtweise wurde allerdings schon oben mit dem Hinweis auf die Interviewanalyse der Lehrendeninterviews, die in Gruber et al. (2006) ausführlich dargestellt wird, widersprochen. Vielmehr reflektieren die oben besprochenen Unterschiede die in Abschnitt 4 abgeleiteten multiplen Zielsetzungen der Textsorte „Seminararbeit““ im sozialen Feld der Universität.

Es zeigt sich allerdings gerade beim professionellen deutschsprachigen Text, dass die im IMRD Modell ganz offensichtliche Problemlösungsstruktur eines wissenschaftlichen Aufsatzes durch die Textstruktur (d. h. die inhaltlich-formale Gliederung) nicht eindeutig signalisiert wird, es fehlt sowohl eine explizite Einleitung als auch eine explizit als solche markierte Diskussion der Ergebnisse. Im größten Teil des Texts werden Ergebnisse präsentiert, ihre Relevanz wird nur im abschließenden Teil ansatzweise thematisiert. Damit realisiert der Aufsatz (bzw. seine inhaltliche Gliederung) eine Mischform dessen, was Rienecker/Stray Jörgenssen (2003) als kontinental-europäisches und anglo-amerikanisches Schreib (und Denk-)Modell einander gegenüber stellen. In seinem formalen Aufbau und den fehlenden metakommunikativen Hinweisen entspricht der Text dem kontinental-europäischen Modell des wissenschaftlichen Schreibens, während er in Bezug auf seinen Fokus auf die Präsentation und Analyse empirischer Daten eher dem anglo-amerikanischen Modell entspricht.

Die Strukturen der komplexen Mittelteile der beiden Texte stimmen in Bezug auf ihre kommunikative Funktion eher überein, obwohl sich ihre Makrostrukturen stärker unterscheiden als die Strukturen der beiden Gesamttexte. In beiden Texten überwiegen auf der Makroebene nicht-argumentative Relationen zwischen den einzelnen Unterkapiteln. Dieses Merkmal wiederum entspricht dem IMRD- Modell insofern als im Resultateteil eher deskriptive Darstellungen von Einzelergebnissen zu erwarten sind, die erst im abschließenden Diskussionsteil argumentativ mit den Fragestellungen in Zusammenhang gebracht werden.

Interessanterweise waren im studentischen Text tendenziell mehr strukturierende und metatextuelle Sequenzen zu finden als im professionellen Text. Das könnte ein Hinweis darauf sein, dass sich der professionelle Autor in einem hohen Maß auf das Vorwissen seiner Leserschaft verlässt und es als nicht notwendig erachtet, explizite textstrukturierende oder 
kohärenzstiftende Signale zu verwenden, während der studentische Autor sichergehen will, dass Aufbau und Struktur seines Texts tatsächlich so rezipiert werden, wie es von ihm intendiert ist. Dieses Ergebnis kann als Hinweis darauf gelten, dass bei einem Fachpublikum (und einem professionellen Schreibenden) ein höheres $\mathrm{Ma} 3$ an inhaltlichem und textsortenbezogenen Wissen vorausgesetzt werden kann, während ein ungeübter Schreiber mehr Aufmerksamkeit darauf verwendet, die von ihm in einem Text intendierten Zusammenhänge explizit und damit nachvollziehbar zu machen.

Der Vergleich der Einleitungen der beiden Texte (und damit eine Untersuchung ihrer Mesound Mikroebene) zeigt, dass das Swalessche Modell mit seiner (in der ursprünglichen Version) vorgegebenen Abfolge von Phasen und Zügen für deutsche Texte eher einen Fundus an möglichen rhetorischen Elementen als ein fixes Textschema zur Verfügung stellt (wobei eingeschränkt werden muss, dass Swales (2004) ebenfalls konzediert, dass die Phasen und Züge, die sein Modell für die Einleitung eines wissenschaftlichen Aufsatzes vorsieht, nicht unbedingt in einer fixierten Reihenfolge realisiert werden müssen). In beiden Einleitungen fanden sich Phasen bzw. Züge, die das Modell vorsieht, allerdings nicht alle und darüber hinaus fanden sich in der professionellen Einleitung Elemente, die das Modell nicht vorsieht.

Beide Texte kommen relativ unmittelbar zur Formulierung des zentralen Anliegens (bzw. Problems), das im Text behandelt wird, ohne lange ein „Territorium“ zu etablieren. In beiden Texten wird das zentrale Anliegen einleitend formuliert und dann nochmals wiederholt, allerdings leitet im professionellen Text diese Wiederholung zur Ankündigung der eigenen Untersuchung über, während im studentischen Text die Wiederholung der Textintention am Ende der Einleitung eher repetitiv (und damit dysfunktional) wirkt. Diese Unterschiede zwischen den beiden Texten weisen eher auf eine mangelnde Textsortenkompetenz des studentischen Autors hin, also auf einen anderen oben erwähnten Unterschied zwischen professionellen und studentischen Autoren. Darüber hinaus ist dieses Resultat auch im Einklang mit der Untersuchung von Pohl (2007), der selbst bei kompetenten studentischen Schreibenden Texteinleitungen fand, die dem Swalesschen Modell nicht bzw. nur in Teilen entsprachen.

Die hier präsentierte qualitative Untersuchung unterstützt auch die einleitend diskutierte Notwendigkeit der internen Differenzierung „großer“ (sprachlicher) in verschiedene „kleine“ (fach- und institutionsspezifische, auf das Ausmaß des Expertenstatus bezogene) Kulturen. Denn gerade in Bezug darauf liefert die vorliegende Analyse reiche Anhaltspunkte für weitere Untersuchungen: so zeigte sich, dass der professionelle Text zwar auf der Makrostruktur dem Swalesschen Modell des problemlösenden Texts eher entsprach als der studentische, auf der inhaltlichen Ebene und in Bezug auf ausgewählte mikrostrukturelle Elemente aber eine Mischform des kontinental-europäischen und des anglo-amerikanischen Schreibmodells (vgl. Rienecker/Stray Jörgenssen 2013) realisierte und außerdem rhetorische Züge aufwies, die einer „konventionellen“ Einleitung eines wissenschaftlichen Aufsatzes nach den Modellannahmen nicht zu erwarten wären.

Demgegenüber zeigte der studentische Text oberflächlich betrachtet stärkere Übereinstimmungen mit dem klassischen Swalesschen Modell, eine nähere Untersuchung der rhetorischen Beziehungen zwischen den einzelnen Phasen und Zügen des Texts zeigte aber schnell, dass diese Ähnlichkeiten eher simuliert als real existent waren. An diesem Ergebnis 
lässt sich auch die Relevanz der Kombination von rhetorisch-sprechhandlungsorientierter Textsortenanalyse mit einer kohärenzorientierten Makrostrukturanalyse demonstrieren.

Die Unterschiede zwischen dem professionellen und dem studentischen Text könnten mit der unterschiedlichen Textsortenkompetenz der beiden Schreibenden insofern erklärt werden, als man behaupten könnte, dass der studentische Autor sich (zumindest oberflächlich gesehen) stärker an einem vorgegebenen Modell orientiert, während der geübte Autor beim Schreiben seine Textintentionen in den Vordergrund stellen und bei seinem Publikum auch ein Maß an Fachwissen und Textsortenkompetenz voraussetzen kann, auf das sich der studentische Autor offenbar nicht verlassen wollte.

Eine genauere Analyse hat aber gezeigt, dass die unterschiedlichen Textstrukturen, die die Analyse aufgewiesen hat, zwar an den beiden letzteren Erklärungsfaktoren ansetzen kann, aber viel stärker auf die unterschiedliche institutionelle Position der beiden Autoren Bezug nehmen muss: der für ein Fachpublikum schreibende professionelle Autor kann sein Publikum unter den Druck einer Vorwissenserwartung setzen, den der studentische Autor sich nicht leisten kann - für ihn ist es relevant seinem Seminarleiter zu beweisen, dass er die gesamte relevante Literatur bearbeitet und integriert hat und ebenso, dass er sich an den im Seminar gestellten Textsortenvorgaben orientiert, auch wenn er sie rhetorisch nicht erfüllen kann.

Soweit eine qualitative Analyse zweier Texte überhaupt generalisierbare Ergebnisse erbringen kann, stützen die eben diskutierten Resultate doch die eingangs formulierte These, dass es sich bei den (Sub-) Textsorten, die unter der Bezeichnung „Forschungsaufsatz“ produziert werden ebenso wie bei den auf ihrer Basis entstehenden Arbeiten fortgeschrittener Studierender um eine „Textsortenkolonie“ untereinander durch ein System von Familienähnlichkeiten verbundener Textsorten handelt. Übereinstimmungen und Unterschiede zwischen den einzelnen Mitgliedern einer solchen Kolonie können - wie die vorhergehende Diskussion zeigt - auf institutionelle Faktoren und daraus resultierende kommunikative Zwecke ebenso wie auf Unterschiede in der jeweiligen Textsortenkompetenz der Schreibenden (und damit auf kognitive Faktoren) zurückgeführt werden.

Die vorliegenden Ergebnisse zeigen damit auch, dass im Bereich der Untersuchung der Rhetorik der interkulturellen Wissenschaftskommunikation noch viele offene Fragen vorhanden sind, die einer Beantwortung harren und dass die Annahme globaler rhetorischer „Schreibkulturen“, die für ganze Sprach- oder Kulturkreise charakteristisch wären, wahrscheinlich den Blick auf die empirischen Daten eher verstellt als erhellt.

\section{Literaturverzeichnis}

Atkinson, Dwight (2004): "Contrasting rhetorics/contrasting cultures: why contrastive rhetoric needs a better conceptualization of culture". Journal of English for Academic Purposes 3/4: 277-289. doi:10.1016/j.jeap.2004.07.002.

Atkinson, Dwight/Sohn, Jija (2013): "Culture from the Bottom Up". TESOL Quarterly 47/4: 669-693. doi:10.1002/tesq.104.

Barton, David/Hamilton, Mary (2000): “Literacy Practices”. In: Barton, David/Hamilton, Mary/Ivanič, Roz (eds.): Situated Literacies: Reading and writing in context. London, Routledge: $7-15$. 
Baßler, Harald (2003): „Russische, deutsche und angloamerikanische Zeitschriftenabstracts der Soziologie: Worin unterscheiden sie sich?“. In: Gruber, Helmut/Menz, Florian/Panagl, Oswald (eds.): Sprache und politischer Wandel. Frankfurt a. M., Lang: 189-212.

Bazerman, Charles (1988): Shaping Written Knowledge. The Genre and Activity of the Experimental Article in Science. Madison, Wis.: The University of Wisconsin Press.

Berkenkotter, Carol/Huckin, Thomas N. (1995): Genre knowledge in disciplinary communication: Cognition / Culture / Power. Hillsdale, NJ/Hove: Erlbaum.

Bhatia, Vijay K. (2004): Worlds of Written Discourse. A Genre-Based View. London: Continuum.

Clark, Romy/Ivanic, Roz (1997): The politics of writing. London: Routledge.

Clyne, Michael (1987): "Cultural Differences in the Organization of Academic Texts. English and German". Journal of Pragmatics 11: 211-247.

Connor, Ulla (2004): "Intercultural rhetoric research: beyond texts". Journal of English for Academic Purposes 3/4: 291-304. doi:10.1016/j.jeap.2004.07.003.

Dressen-Hammouda, Dacia (2008): "From novice to disciplinary expert: Disciplinary identity and genre mastery". English for Specific Purposes 27/2: 233-252. doi:10.1016/j.esp.2007.07.006.

Ebster, Claus/Stalzer, Lieselotte (2003): Wissenschaftliches Arbeiten für Wirtschafts- und Sozialwissenschaftler. 4. Auflage, Wien: Facultas.

Loi, Chek Kim (2010): "Research article introductions in Chinese and English: A comparative genre-based study". Journal of English for Academic Purposes 9/4: 267-279. doi:10.1016/j.jeap.2010.09.004.

Fritz, Gerd (2014): „Texttheorie und textlinguistische Annotation von Korpora: Bemerkungen zur Rhetorical Structure Theory“. Zeitschrift für germanistische Linguistik 42/2: 163-195. doi:10.1515/zgl-2014- 0012.

Galtung, Johan (1985/1981): „Struktur, Kultur und intellektueller Stil. Ein vergleichender Essay über sachsonische, teutonische, gallische und nipponische Wissenschaft". In: Wierlacher, Alois (ed.): Das Fremde und das Eigene. Prolegomena zu einer interkulturellen Germanistik. München, Iudicium: 151-193.

Gnutzmann, Claus/Lange, Regina (1990): „Kontrastive Textlinguistik und Fachsprachenanalyse“. In: Gnutzmann, Claus (ed.): Kontrastive Linguistik. Frankfurt a. M., Lang: 85-117.

Gnutzmann, Claus/Rabe, Frank (2014): “Theoretical subtleties' or 'text modules'? German researchers' language demands and attitudes across disciplinary cultures". Journal of English for Academic Purposes 13: 31-40. doi:10.1016/j.jeap.2013.10.003.

Gray, Bethany E. (2011): Exploring academic writing through corpus linguistics: When discipline tells only part of the story. PhD Dissertation, Northern Arizona University.

Gruber, Helmut (2007): „Wissenschaftskulturen und studentisches Schreiben - die Ergebnisse einer diskursanalytischen Studie“. In: Doleschal, Ursula/Hoffmann, Edgar/Reuther, Tilman (eds.): Sprache und Diskurs in Wirtschaft und Gesellschaft - Interkulturelle Perspektiven. Frankfurt a. M., Lang: 43-60.

Gruber, Helmut (2013): „Kulturen des studentischen Schreibens. Institutionelle, disziplinspezifische und sprachliche Aspekte“. In: Vogt, Irene et al. (eds.): Fremdes wahrnehmen, aufnehmen, annehmen - Studien zur deutschen Sprache und Kultur in Kontaktsituationen. Bonn, Lang: 323-343. (=Bonner Romanistische Arbeiten 109). 
Gruber, Helmut (2014): "Signaling coherence in Austrian students' seminar papers: macroand micro-structural cues". In: Gruber, Helmut/Redeker, Gisela (eds.): The pragmatics of Discourse Coherence. Theories and Applications. Amsterdam, Benjamins: 267-291. (= Pragmatics and Beyond New Series 254).

Gruber, Helmut/Muntigl, Peter (2005): “Generic and rhetorical structures of texts: Two sides of the same coin?". Folia Linguistica 39/1: 75-113.

Gruber, Helmut et al. (2006): Genre, Habitus und Wissenschaftliches Schreiben - eine empirische Untersuchung studentischer Texte. Wien: LIT.

Holliday, Adrian R. (1999): "Small cultures". Applied Linguistics 20/2: 237-264. doi:10.1093/applin/20.2.237.

Hyland, Ken (2009): Academic Discourse: English in a Global Context. London: Continuum.

Hyland, Ken (2013): Writing in the university: education, knowledge and reputation. Language Teaching 46/1: 53-70. doi:10.1017/S0261444811000036.

Ivanic, Roz (1998): Writing and Identity: The discoursal construction of writer identity. Amsterdam: Benjamins.

Jones, Carys/Turner, Joan/Street, Brian (eds.) (1999): Students Writing in the University. Cultural and Epistemological Issues. Amsterdam: Benjamins.

Kaplan, Robert B. (1966): "Cultural thought patterns in intercultural education". Language Learning 16: 1-20.

Lea, Mary (1999): "Academic Literacies and Learning in Higher Education. Constructing knowledge through texts and experience". In: Jones, Carys/Turner, Joan/Street, Brian (eds.): Students writing in the university. Cultural and epistemologicial issues. Amsterdam, Benjamins: 103-125.

Lillis, Theresa (2001): Student Writing. Access, Regulation, Desire. London: Routledge.

Mann, William/Thompson, Sandra (1986): "Relational Propositions in Discourse". Discourse Processes 9/1: 57-90.

Mann, William/Thompson, Sandra (1988): "Rhetorical Structure Theory: Towards a functional theory of text organization". Text 8/3: 243-281.

Mann, William/Thompson, Sandra (eds.) (1992): Discourse Descriptions. Amsterdam: Benjamins.

Mann, William/Matthiessen, Christian/Thompson, Sandra (1992): "Rhetorical Structure Theory and Text Analysis". In: Mann, William/Thompson, Sandra (eds.): Discourse Descriptions. Amsterdam, Benjamins: 39-77.

Mathiessen, Christian (2014): "Registerial cartography: context-based mapping of text types and their rhetorical-relational organization". 28th Pacific Asia Conference on Language, Information and Computation. Hong Kong: 5-26.

Mauranen, Anna/Perez-Llantada, Carmen/Swales, John M. (2010): “Academic Englishes: A standardized knowledge?". In: Kirkpatrick, Andy (ed.): The Routledge Handbook of World Englishes. Oxon/New York/Milton Park, Routledge: 634-652.

Nippa, Michael (1997): „Erfolgsfaktoren organisatorischer Veränderungsprozesse in Unternehmen. Ergebnisse einer Expertenbefragung.“ In: Nippa, Michael/Scharfenberg, Heinz (Hrsg): Implementierungsmanagement. Wiesbaden, Gabler: 21-57.

Pohl, Thorsten (2007): Studien zur Ontogenese wissenschaftlichen Schreibens. Tübingen: Niemeyer. 
Redeker, Gisela/Gruber, Helmut (2014): "Introduction: The Pragmatics of discourse coherence". In: Gruber, Helmut/Redeker, Gisela (eds.): The Pragmatics of Discourse Coherence. Theories and Applications. Amsterdam/Philadelphia, Benjamins: 1-20.

Rienecker, Lotte/Stray Jörgensen, Peter (2003): "The (im)possibilities in teaching university writing in the Anglo-American tradition when dealing with continental student writers". In: Björk, Lennart/Bräuer, Gerd/Rienecker, Lotte/Stray Jörgensen, Peter (eds.): Teaching Academic Writing in European Higher Education. Dordrecht, Kluwer: 101-113.

Sanders, Ted/Schilperoord, Joost (2008): "Text structure as a window on the cognition of writing: How text analysis provides insights in writing products and writing processes". In: MacArthur, Charles A./Graham, Steve/Fitzgerald, Jill (eds.): Handbook of Writing Research. New York, Guilford Press: 386-402.

Speyer, Augustin/Fetzer, Anita (2014): "The coding of discourse relations in English and German argumentative discourse". In: Gruber, Helmut/Redeker, Gisela (eds.): The pragmatics of Discourse Coherence. Theories and Applications. Amsterdam, Benjamins: 87-121.

Stede, Manfred (2007): Korpusgestützte Textanalyse: Grundzüge der Ebenen-orientierten Textlinguistik. Tübingen: Narr Francke Attempto.

Swales, John (1990): Genre Analysis. Cambridge: Cambridge University Press.

Swales, John (2004): Research Genres: Explorations and Applications. Cambridge: Cambridge University Press.

Taylor, Gordon/Chen, Tingguang (1991): "Linguistic, Cultural, and Subcultural Issues in Contrastive Discourse Analysis: Anglo-American and Chinese Scientific Texts". Applied Linguistics 12/3: 319-336. doi:10.1093/applin/12.3.319.

van der Vliet, N.,/Redeker, Gisela (2014): "Explicit and implicit coherence relations in Dutch texts". In: Gruber, Helmut/Redeker, Gisela (eds.): The Pragmatics of Discourse Coherence. Theories and Applications. Amsterdam/Philadelphia, Benjamins: 23-53.

Wittgenstein, Ludwig (1971): Philosophische Untersuchungen. Frankfurt a. M.: Suhrkamp.

\section{Anhang: Relationsdefinitionen (geordnet nach ihrem Auftreten in den Diagrammen im} Text)

Abb. 2 (Makrostruktur IMRD Schema):

- Solutionhood: "N is a solution to the problem presented in $\mathrm{S}$ "

- Circumstance: "S sets a framework in the subject matter within which $\mathrm{R}$ is intended to interpret N"

- Interpretation: "S relates $\mathrm{N}$ to a framework of ideas not involved in $\mathrm{N}$ itself and not concerned with W's positive regard" (d. h. es handelt sich um keine positive Bewertung des Inhalts von $\mathrm{N}$ durch den/die Schreibende sondern um eine theoretische Reflexion über den Inhalt von N; HG)

Abb. 4 (Makrostruktur professioneller deutschsprachiger Text):

- Elaboration: "S presents additional detail about the situation or some element of subject matter which is presented in $\mathrm{N}$ or inferentially accessible in $\mathrm{N}$ in one or more of the ways listed below. In the list, if $\mathrm{N}$ presents the first member of any pair, then $\mathrm{S}$ includes the second: 
○ set :: member

○ abstraction :: instance

○ whole :: part

○ process :: step

○ object :: attribute

○ generalization :: specific“"

- conjunction: „The items are conjoined to form a unit in which each item plays a comparable role"

Abb. 6 (Makrostruktur des studentischen Texts):

- (Content) preparation: "S precedes $\mathrm{N}$ in the text; $\mathrm{S}$ tends to make $\mathrm{R}$ more ready, interested or oriented for reading N" - "content preparation" (im Kontrast zu "structural preparation") bezieht sich auf eine inhaltliche Vorbereitung der Leser auf den folgenden Nukleus, während „structural preparation“ die Leser auf die Struktur des folgenden Nukleus vorbereitet.

- Background: "S increases the ability of R to comprehend an element in N."

- Summary: "S presents a restatement of the content of N, that is shorter in bulk."

Abb. 7 und 8 (Kohärenzstrukturen der Einleitungen von professionellem und studentischem Text):

- Restatement (Bsp. 4): „on $\mathrm{N}+\mathrm{S}$ : $\mathrm{S}$ restates $\mathrm{N}$, where $\mathrm{S}$ and $\mathrm{N}$ are of comparable bulk; $\mathrm{N}$ is more central to $\mathrm{W}$ 's purposes than $\mathrm{S}$ is."

- Restatement multinuclear (Bsp. 5): "An item is primarily a reexpression of one linked to it; the items are of comparable importance to the purposes of W"

- Antithesis: „N and $\mathrm{S}$ are in contrast (see the Contrast relation); because of the incompatibility that arises from the contrast, one cannot have positive regard for both of those situations; comprehending $\mathrm{S}$ and the incompatibility between the situations increases R's positive regard for N."

- Concession: "W acknowledges a potential or apparent incompatibility between $\mathrm{N}$ and $\mathrm{S}$; recognizing the compatibility between $\mathrm{N}$ and $\mathrm{S}$ increases R's positive regard for N."

- Justify: "R's comprehending S increases R's readiness to accept W's right to present N." 